\title{
INFLUENCE OF TIME-DEPENDENT POWER DISSIPATIONS ON THE AGEING BEHAVIOR OF THICK-FILM RESISTORS
}

\author{
G. DE MEY, E. BOONE AND G. DE TOLLENARE \\ Laboratory of Electronics, University of Ghent, Sint Pietersnieuwstraat 41, 9000 Ghent, Belgium
}

(Received June 10, 1992; in final form January 13, 1993)

Reliability experiments on hybrid circuits are usually carried out by accelerated ageing test. The circuits or the components under test are stored at an elevated ambient temperature and the change of their electric properties is controlled at regular times. The situation becomes entirely different if the temperature rise is due to the power dissipation in the component under test and not caused by external means. In electronic circuits, power dissipations are expressed by a mean value, whereas the actual situation is generally a time-dependent function. The temperature will then also be time dependent. Therefore, ageing tests on thick film resistors will be presented in this contribution. Resistors are submitted to a DC power source and a pulse shaped one. Different ageing characteristics are observed.

\section{INTRODUCTION}

It is a well known phenomenon that ageing of electronic components is accelerated at higher temperatures [1]. For high-precision circuits involving trimmed resistors, the variation of the resistance values should be kept within a narrow limit. Otherwise, a good circuit performance cannot be guaranteed for the expected lifetime of the circuit. Thermal management then becomes necessary to keep temperatures sufficiently low $[2,3,4,5]$.

On the other hand, ageing experiments are carried out at elevated temperatures in order to accelerate the test [1]. The devices under test are then placed in a hermetically closed vessel with a constant internal temperature and humidity: the ambient conditions are constant with respect to time. If the ambient temperature is kept within very narrow limits, high-precision measurements can seriously reduce the measuring time [6].

In this contribution, ageing experiments will be presented on screen-printed thick-film resistors. The resistors are only warmed up by their own power dissipations, which is certainly a better approach to the actual situation occurring in operational hybrid circuits. One may wonder how this approach can give different results. If the resistor would have been stored at the same temperature without any internal dissipation, the same ageing behavior can be expected. No arguments can be put forward why the ageing should be different. We disregard here special effects due to high current densities or electric field strengths. The situation becomes entirely different if time-dependent powers are involved. The power dissipation in 


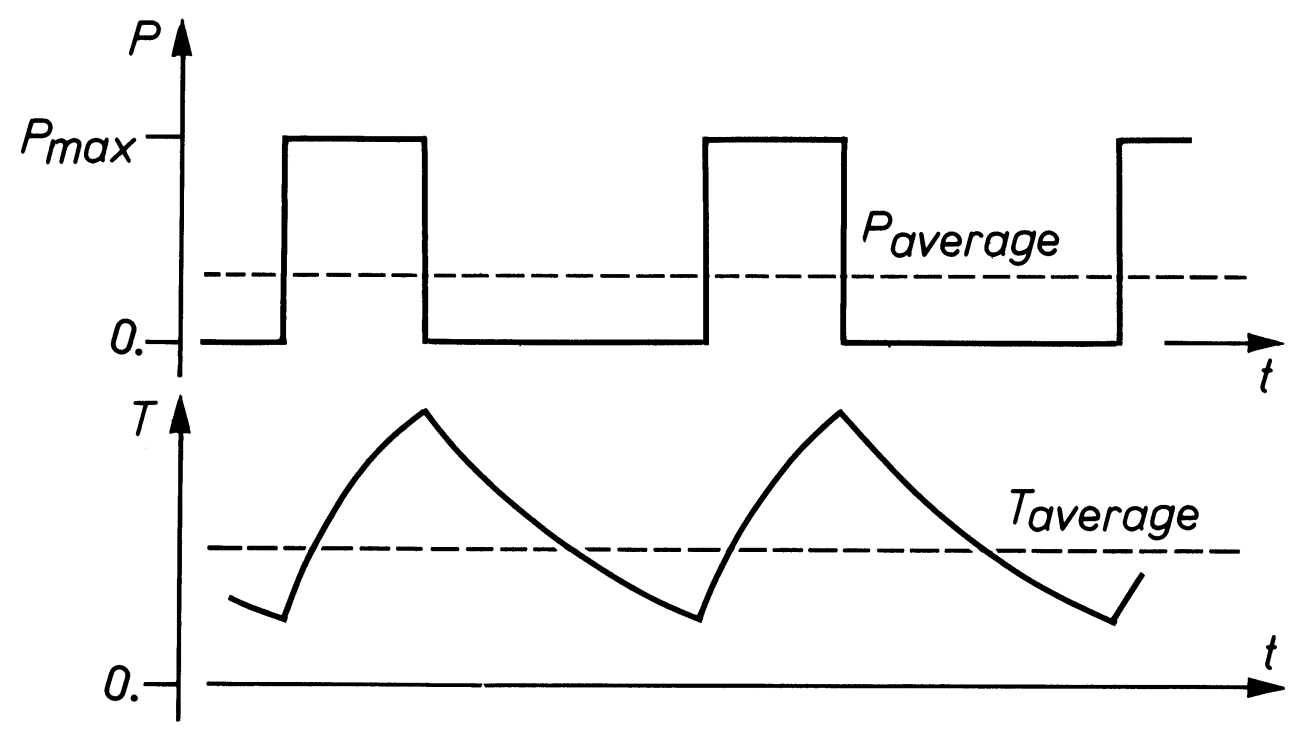

FIGURE 1 Power and temperature of a thick film resistor.

thick-film resistors or other electronic components is described by its time average value. It has been calculated theoretically that fast thermal transients can occur in hybrid circuits, especially if the thermal transient on the heating resistor is concerned [7]. Using a new infrared thermography technique, a ramp of $2 \mathrm{~K} / 30 \mu \mathrm{sec}$ has been reported on a thick-film resistor [8]. If a time varying voltage is applied across a resistor, the temperature will no longer be constant but fluctuates around the mean value. Fig. 1 shows a possible situation if periodic pulses are applied. The maximum temperature can be much higher than the mean value, usually defined as "the" temperature. Most temperature sensors only give the mean value. Having a non-negligible thermal mass or capacity, they act as a low-pass filter for the temperature signal.

The question now is which temperature is responsible for the ageing of the resistor: is it the maximum, the mean temperature or should one consider the temperature as a function of time $T(t)$. Therefore, two ageing experiments were carried out. A constant power was applied to a first set of resistors and a periodic pulse train to a second set. The variation of the resistance values were measured for a long period.

\section{THEORY}

Although the theory presented here is not based on well developed physical principles, it will give us some indications of what can be expected in the case of a time varying temperature.

Under a constant temperature, the relative variation $\Delta R / R$ of the resistance value is expressed by the well known Arrhenius relation:

$$
\frac{\Delta \mathrm{R}}{\mathrm{R}}=\operatorname{Aexp}\left(-\frac{\mathrm{E}_{\mathrm{A}}}{\mathrm{kT}}\right)
$$


where:

- $\mathrm{E}_{\mathrm{A}}$ : Activation energy of the ageing process

- k: Boltzmann constant

- T: Absolute temperature

If the temperature varies with time, one gets, assuming (1) being valid for any time increment dt:

$\frac{\Delta \mathrm{R}}{\mathrm{R}}=\mathrm{A} \frac{1}{\mathrm{t}} \int_{0}^{\mathrm{t}} \exp \left(-\frac{\mathrm{E}_{\mathrm{A}}}{\mathrm{kT}\left(\mathrm{t}^{\prime}\right)}\right) \mathrm{dt}^{\prime}$

We consider now the special case where the temperature $\mathrm{T}$ varies sinusoidally around a mean value $T_{0}$ :

$T(t)=T_{0}+T_{1} \cos (\omega t)$

$T$ in (1) being an absolute temperature, the amplitude $T_{1}$ in (3) will be much smaller than $\mathrm{T}_{0}$. Some mathematical simplifications can then be carried out after inserting (3) in (2):

$$
\begin{aligned}
\frac{\Delta R}{R} & =\frac{A}{t} \int_{0}^{t} \exp \left(-\frac{E_{A}}{k\left(T_{0}+T_{1} \cos \omega t^{\prime}\right)}\right) d^{\prime} \\
& =\frac{A}{t} \int_{0}^{t} \exp \left(-\frac{E_{A}}{k T_{0}}\left(1-\frac{T_{1}}{T_{0}} \cos \omega t^{\prime}\right)\right) d^{\prime} \\
& =A \exp \left(-\frac{E_{A}}{k T_{0}}\right) \frac{1}{t} \int_{0}^{t} \exp \left(\frac{E_{A} T_{1}}{k T_{0}^{2}} \cos \omega t^{\prime}\right) d^{\prime}
\end{aligned}
$$

For a periodic temperature variation like (3), it is sufficient to limit the integration time in (4) to one period or:

$$
\begin{aligned}
& \frac{\Delta R}{R}=\operatorname{Aexp}\left(-\frac{E_{A}}{k T_{0}}\right) \frac{1}{\pi} \int_{0}^{\pi} \exp \left(\frac{E_{A} T_{1}}{k T_{0}^{2}} \cos \alpha\right) d \alpha \\
&=A \exp \left(-\frac{E_{A}}{k T_{0}}\right) I_{0}\left(\frac{E_{A} T_{1}}{k T_{0}^{2}}\right)
\end{aligned}
$$

where $I_{0}$ is the modified Bessel function of order zero [9].

Fig. 2 shows a plot of the function $I_{0}(x)$. For a typical value $E_{a} / k=8120$ [1], a mean temperature $T_{0}=400 \mathrm{~K}$ and an amplitude $T_{1}=20 \mathrm{~K}$, we obtain $\mathrm{x}=\mathrm{E}_{\mathrm{A}} \mathrm{T}_{1} /$ $\mathrm{kT}_{0}^{2}=1.015$. In Fig. 2 one finds the ageing will proceed 1.27 times faster compared to a constant temperature ageing. This simple theory presented here provides us a good indication that a non-negligible increase of the ageing rate can be expected even for a moderate temperature fluctuation around a mean value. 


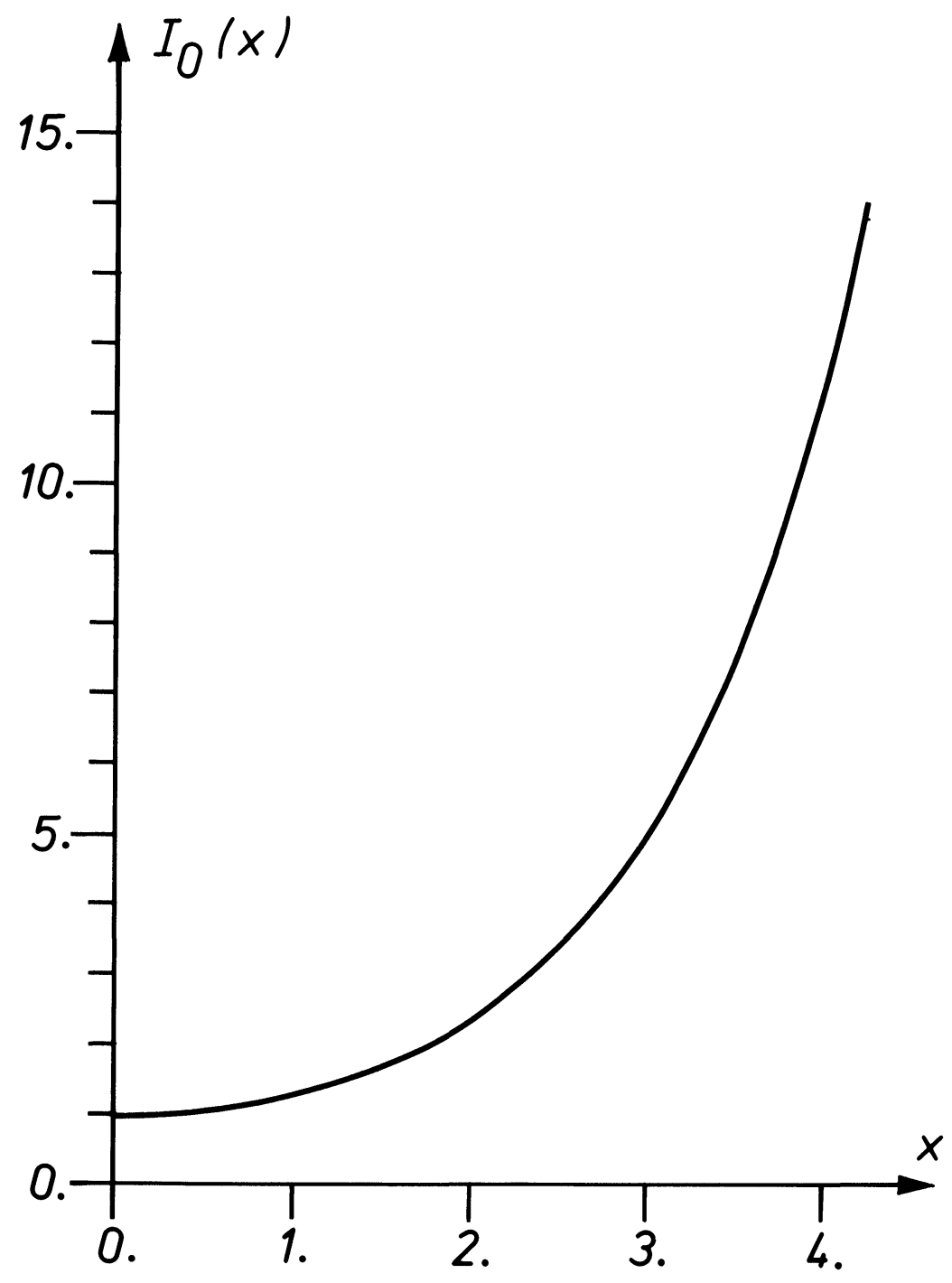

FIGURE 2 Modified Bessel function $\mathrm{I}_{0}(\mathrm{x})$.

\section{EXPERIMENTAL RESULTS}

Ageing experiments were carried out on 4 sets (A, B, C and D) of screen-printed resistors. All samples were made during the same printing and sintering process. The nominal resistance values were about $110 \Omega$. Each set contained 5 resistors so that a total number of 20 resistors were investigated.

The 5 resistors of set A were exposed to a constant DC voltage, regulated such that 1 Watt was dissipated in each of them. At regular times the resistance values were measured. Fig. 3 represents the mean values of the 5 data as a function of time. All results are normalised to the initial value measured at the beginning of 


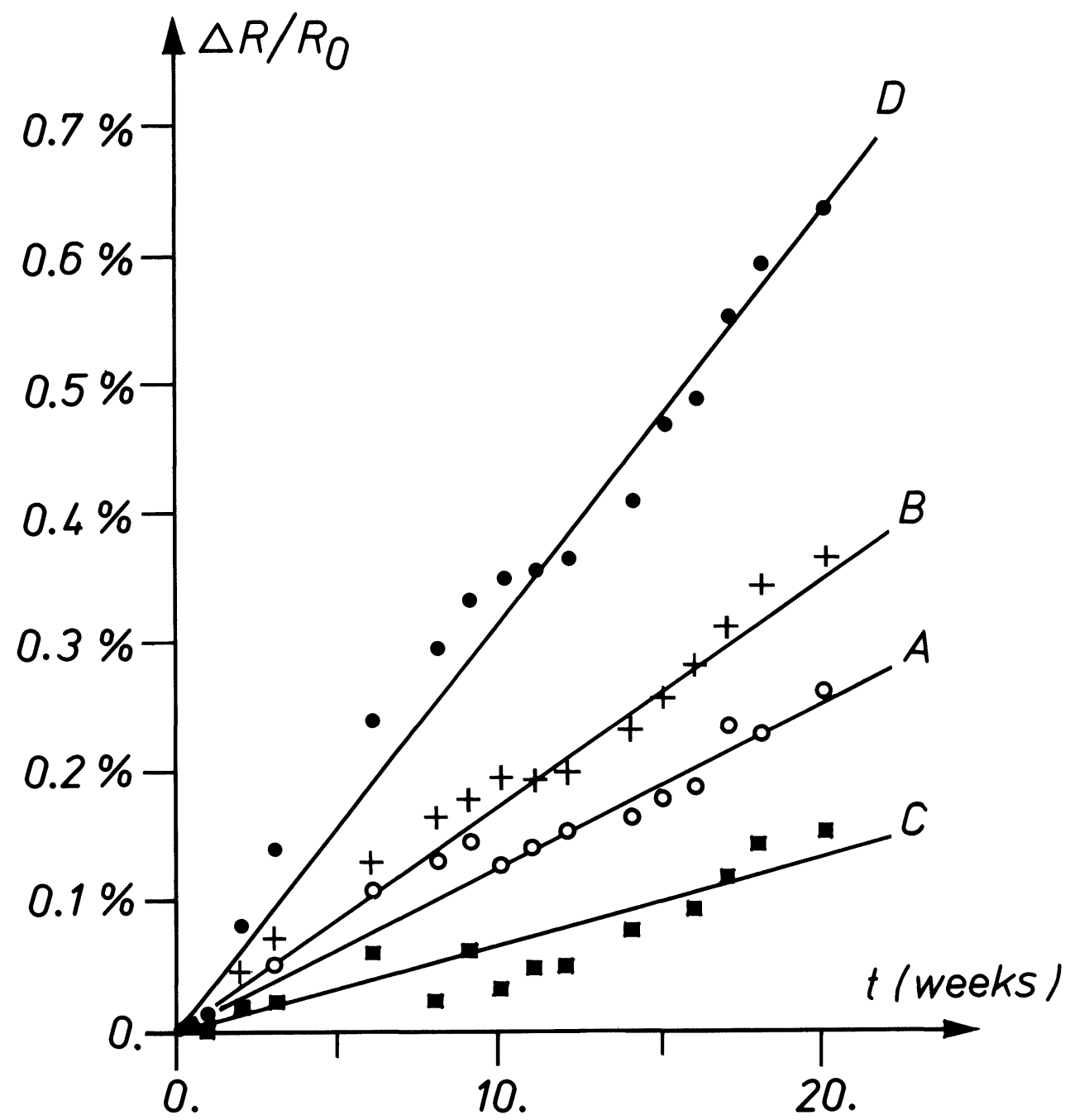

FIGURE 3 Ageing behaviour of 4 set of thick film resistors. A: DC power and air cooling. B: pulse power and air cooling. C: DC power and liquid cooling. D: pulse power and liquid cooling.

the experiment. A change in resistance value of $0.25 \%$ is observed after 20 weeks. The resistors of the B set were exposed to a pulse shaped power. Pulses of $6 \mathrm{Watt}$ peak were applied with a duty ratio of 0.1 , which means that the mean power was only 0.6 Watt. Pulses were applied with a frequency of $0.5 \mathrm{~Hz}$, or one pulse of 200 msec every 2 seconds. As can be seen on Fig. 3, the ageing of set B is going faster even with a smaller mean power. A better comparison between $A$ and $B$ can be made if the results of $A$ are reduced by 0.6 . The ageing of $B$ is then found to be 2.3 times faster. The reason why $\mathrm{B}$ was done with a lower average value was due to the voltage limit for the resistors pastes. For the sets A and B, all substrates were placed vertically and cooled by natural convection. 
The experiments done on the sets $\mathrm{C}$ and $\mathrm{D}$ were similar to the experiments $\mathrm{A}$ and $B$ except the resistors were cooled by immersing the substrates in an inert fluorocarbon liquid. It has been proven that these liquids have no influence on the electric properties of thick film resistors [10]. In the case of a DC power supply, the ageing of the immersed resistors (curve $C$ on Fig. 3 ) is very small. This result is not surprising because the liquid provides a better cooling, resulting in a lower temperature. If pulses are applied (curve D of Fig. 3) an unexpected rapid ageing is observed. Several reasons can be put forward to explain this unusual behavior. At first, due to mechanical inertia, liquids are not as effective for cooling if time varying temperatures are involved. Secondly, during the pulses the maximum resistor temperature reached the boiling point of the liquid. Microscopically, the formation of a boiling bubble can be seen as an explosion. This cavitation effect may damage the resistors surface. It is then quite obvious that an increased ageing has to be observed.

\section{CONCLUSION}

In this contribution, some ageing experiments on thick film resistors have been presented. The resistors were only heated by their own power dissipations to accelerate ageing. Both continuous and time-varying powers have been applied. It was observed experimentally and explained theoretically that time-dependent powers and, hence, time-dependent temperature create faster ageings even when the mean power is not greater than in the continuous case.

\section{REFERENCES}

1. N. Sinnadurai, "Handbook of microelectronics packaging and interconnection technologies," Electrochemical Publications, Ayr, Scotland, 1985, pp. 154-160.

2. D.J. Dean, "Thermal design of electronic circuit boards and packages," Electrochemical Publications, Ayr, Scotland, 1985.

3. G. Ellison, "Thermal computations for electronic equipment," Van Nostrand, New York, 1984.

4. R.T. Howard (ed.), "Thermal management concepts in microelectronic packaging," ISHM Technical Monograph, 6984-003, Silver Spring, 1984.

5. A. Bar-Cohen and A.D. Kraus (eds.), "Advances in thermal modelling of electronic components and systems," Hemisphere Publications, New York, 1988.

6. L. De Schepper, W. De Ceuninck, H. Stulens, L. Stals, R. Vandenberghe, and S. Demolder, "A new approach to the study of the intrinsic ageing kinetics of thick film resistors," Hybrid Circuits, 1990 , vol. 23, p. 5-13.

7. M. Driscart, G. De Mey and L. Rottiers, "Simulation of the transient thermal behaviour in hybrid circuits," Proceedings 6th European Hybrid Microelectronics conference, Bournemouth 3-5/06/ 87, p. 212-217.

8. G. De Mey, E. Boone, W. De Smet, G. Colpaert, G. Nachtergaele and S. Demolder, "Measuring thermal properties in thin multilayer structures," Proceedings 8th European Hybrid Microelectronics conference, Rotterdam, 29-31/05/91, p. 360-367.

9. M. Abramowitz and I. Stegun, "Handbook of mathematical functions," Dover, New York, 1968, p. 376.

10. G. De Mey and L. De Vrieze, "Cooling of hybrid circuits with fluorocarbon liquids," Hybrid Circuits, 1989, vol. 19, p. 76-77. 

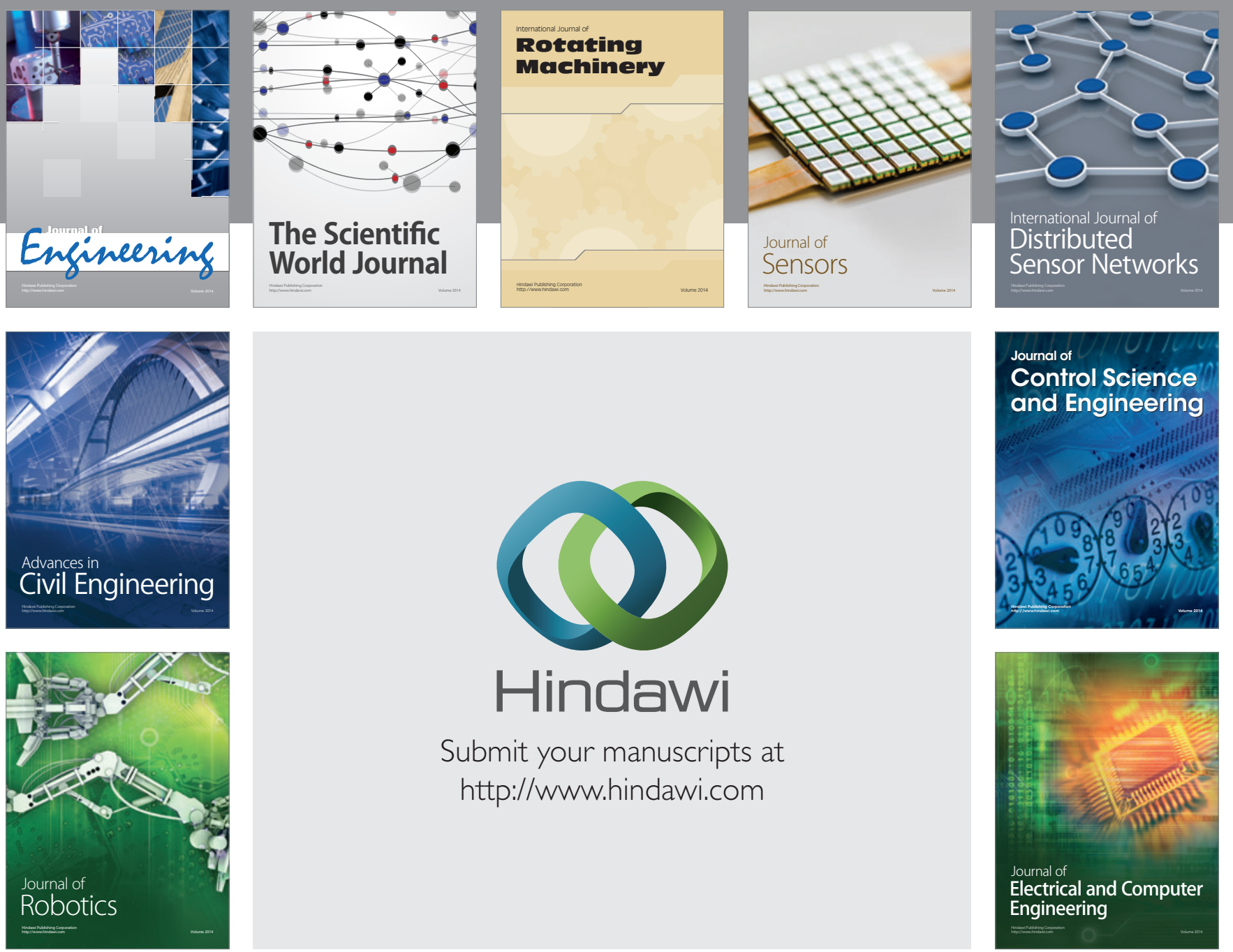

Submit your manuscripts at

http://www.hindawi.com
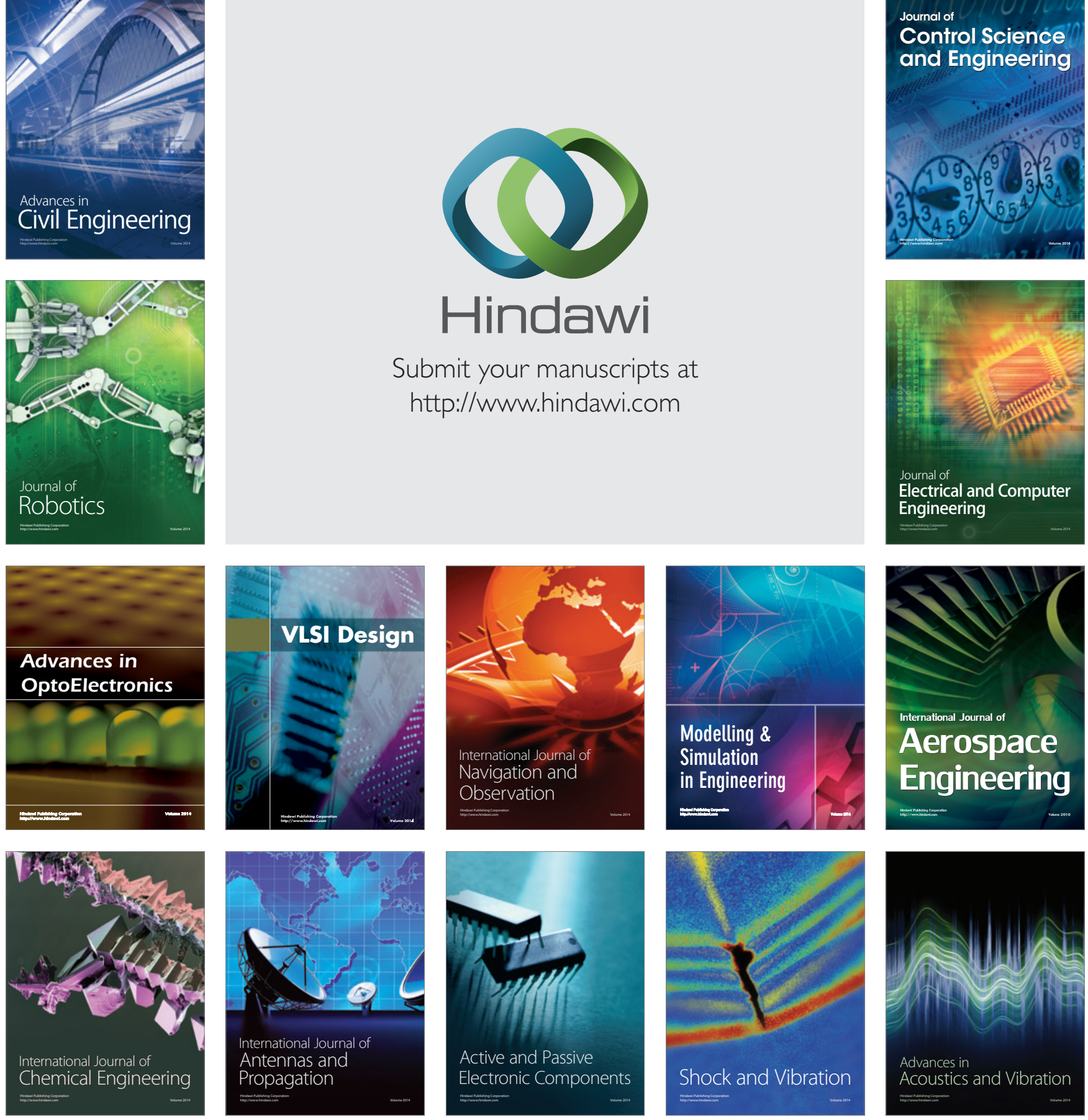